\title{
SOME BOUNDARY VALUE PROBLEMS FOR LINEAR DIFFERENTIAL SYSTEMS ${ }^{1}$
}

\author{
W. J. COLES
}

1. Introduction. Let $A(t)$ and $f(t)$ be $n \times n$ and $n \times 1$ matrices, respectively, continuous on an interval $[a, b]$. In $[1]$, J. B. Garner and L. P. Burton consider the boundary value problems

$$
y^{\prime}=A y+f, \quad y_{i}(a)=\beta_{i}(1 \leqq i<n), \quad y_{n}(b)=\beta_{n}
$$

and

(2) $y^{\prime}=A y+f, \quad y_{1}(a)=\beta_{1}, \quad y_{i}(c)=\beta_{i}(1<i<n, a<c<b)$,

$$
y_{n}(b)=\beta_{n} \text {, }
$$

and prove:

TheOREM A. If, for each $i$ and $j(1 \leqq i \leqq n, 1 \leqq j \leqq n, i \neq j), a_{i j} a_{i n} a_{j_{n}}>0$ and $a_{i n} a_{n i}>0$ on $[a, b]$, the problem (1) has a unique solution;

THEOREM B. Under certain conditions on $A(t)$, too lengthy to give here, the problem (2) has a unique solution.

The authors note that Theorem $A$ has a dual in which the roles of $a$ and $b$ are interchanged, provided that $a_{i j} a_{i n} a_{j n}<0$ is assumed.

The purpose here is to obtain theorems corresponding to Theorem A and its dual, with considerably less restriction on $A(t)$, and to use these results to obtain as a direct consequence a theorem corresponding to Theorem $\mathrm{B}$.

2. The two-point problem. As usual, we rephrase the problem in terms of the homogeneous system. Let $N$ be fixed $(1 \leqq N \leqq n)$; let $Q=\left(\delta_{i N} \delta_{j N}\right)$, and let $P=E-Q$ ( $E$ being the $n \times n$ identity); let $\beta=\operatorname{col}\left(\beta_{i}\right)$. Let $z(t)$ be a solution of $y^{\prime}=A y+f$ which does not satisfy

$$
y^{\prime}=A y+f, \quad P y(a)+Q y(b)=\beta .
$$

If $X$ is any nonsingular solution of $X^{\prime}=A X$, the general solution of $y^{\prime}=A y+f$ can be written in the form $X c+z$, and our boundary condition reduces to

$$
[P X(a)+Q X(b)] \cdot c=\beta-P z(a)-Q z(b) \neq 0 .
$$

Thus (3) has a unique solution if and only if the equation $X^{\prime}=A X$

Received by the editors September 6, 1962.

1 This work was partially supported by National Science Foundation Research Grant G23719. 
has a nonsingular solution for which $P X(a)+Q X(b)$ is nonsingular. We may assume that $X(a)=E$; our condition is then that $x_{N}(b) \neq 0$ if $x^{\prime}=A x$ and $x_{i}(a)=\delta_{i N}(1 \leqq i \leqq n)$.

For convenience we list the following conditions and definitions.

(0) $a_{i i}(t) \leqq 0(1 \leqq i \leqq n)$.

(I) For $N$ fixed $(1 \leqq N \leqq n)$, there exist $K \neq N(1 \leqq K \leqq n)$ and $m_{K}\left(1 \leqq m_{K} \leqq n-1\right)$ such that no product $a_{K_{j_{1}}}\left(t_{0}\right) a_{j_{1} j_{2}}\left(t_{1}\right) \cdots a_{j_{m} N}\left(t_{m}\right)$, with at most $m_{K}+1$ factors, changes sign on $a \leqq t_{i} \leqq b(0 \leqq i \leqq m)$, each such product has the same sign, and one such product, with at most $m_{K}$ factors, is nonzero at $t=a$. Let $s_{K N}$ be 1 or -1 , according as this last product is positive or negative at $t=a$; let $s_{N N}=1$.

(II) For $N$ fixed, (I) holds for each $K \neq N ; s_{K N} a_{N K}(t) \geqq 0$; the $m_{K}$ 's may be taken equal.

(III) $x^{\prime}=A x$ and $x_{i}(a)=\delta_{i N}(1 \leqq i \leqq n)$.

LEmMA 1. If $x^{\prime}=A x$ and (0) holds, and if, for a fixed $j_{i}, \sigma_{i}$ is the set of integers including $1, \cdots, n$, but excluding $j_{i}$, then

$$
\text { (4i) } \begin{aligned}
x_{j_{0}}\left(t_{0}\right)= & x_{j_{0}}(a)+\sum_{j_{1} \in \sigma_{0}} \int_{a}^{t_{0}} a_{j_{0} j_{1}}\left(t_{1}\right) x_{j_{1}}\left(t_{1}\right) d t_{1} ; \\
\text { (4ii) } x_{j_{0}}\left(t_{0}\right)= & x_{j_{0}}(a)+\sum_{h=1}^{m} \sum_{j_{1} \in \sigma_{0}} \ldots \sum_{j_{h} \in \sigma_{h-1}} \int_{a}^{t_{0}} d t_{1} \ldots \\
& \cdot \int_{a}^{t_{h-2}} d t_{h-1} \int_{a}^{t_{h-1}} x_{j_{h}}(a) \prod_{i=0}^{h-1} a_{j_{i} j_{i+1}}\left(t_{i+1}\right) d t_{h} \\
& +\sum_{j_{1} \in \sigma_{0}} \ldots \sum_{j_{m+1} \in \sigma_{m}} \int_{a}^{t_{0}} d t_{1} \ldots \\
& \cdot \int_{a}^{t_{m-1}} d t_{m} \int_{a}^{t_{m}} x_{j_{m+1}}\left(t_{m+1}\right) \prod_{i=0}^{m} a_{j_{i} j_{i+1}}\left(t_{i+1}\right) d t_{m+1}, \quad m \geqq 1 .
\end{aligned}
$$

Proof. Integration of $x_{j_{0}}^{\prime}$ gives (4i) and, in fact, a similar expression for each $x_{j_{i}}$. Substituting these expressions into the right-hand side of (4i), and continuing the process, gives (4ii).

LEMMA 2. If (0), (I), and (III) hold, there is a $\delta>0$ such that $s_{K N} x_{K}(t)$ $>0$ on $(a, a+\delta)$.

Proof. In (4), let $j_{0}=K$ and $j_{m+1}=N$. By (III), the first term in each of (4i) and (4ii) is zero. Since all possible products with $m+1$ factors and of the type in (I) occur in the last set of terms in (4i) or (4ii), proper choice of $m$ will cause to appear a product involving $x_{N}$ which is nonzero at $a$. This term, and indeed all terms involving $x_{N}$, 
have the sign of $s_{K N}$. All other terms in the last set are zero at $a$; all nonzero terms in the second set of terms in (4ii) involve $x_{N}(a)$, and so have the sign of $s_{K N}$. Hence, for small positive $\delta, s_{K N} x_{K}(t)>0$ on $(a, a+\delta)$.

Corollary 1. If (0), (II) and (III) hold, there is a $\delta>0$ such that (5)

$$
s_{i N} x_{i}(t)>0 \quad(1 \leqq i \leqq n)
$$

on $(a, a+\delta)$.

LEMma 3. If (0), (II) and (III) hold, then (5) holds on $(a, b]$.

Proof. By Corollary 1 , there is a $\delta$ such that $0<\delta<b-a$ and for which (5) holds on $(a, a+\delta)$. Let $a<c<a+\delta$, and let $P(t)=$ $x_{1}(t) \cdots x_{n}(t)$. Then

$$
P(t)=P(c) \exp \int_{c}^{t} \sum_{k, j=1 ; k \neq j}^{n} a_{k j} x_{j} / x_{k} d t
$$

on $(c, a+\delta)$. Now, for each $k$ and $j$ such that $k \neq j$, we have $a_{k j} x_{j} / x_{k}$ $\geqq 0$. Indeed, if $k=N$ or $j=N$ this follows from the condition $s_{i N} a_{N i}(t)$ $\geqq 0$ in (II) and from the conclusion of Lemma 2 . If $j \neq N$ and $k \neq N$, let $m$ be the common value of the $m_{i}$ 's in (II). There is a product $P_{j N}$ of the form in (I) (with $K=j$ ), with at most $m$ factors, such that $s_{j N} P_{j N}(a)>0$. Since $a_{k j} P_{j N}$ is a product like those in (I), with at most $m+1$ factors, we have $s_{k N} a_{k j} P_{j N} \geqq 0$. Hence we can write $a_{k j} x_{j} / x_{k}$ $=\left[a_{k j} P_{j N}(a) / x_{k}\right] \cdot\left[x_{j} / P_{j N}(a)\right]$, with each factor non-negative. Thus the statement is verified. From this, $|P(t)| \geqq|P(c)|$ on $(c, a+\delta)$. Since the inequality must hold even for $t=a+\delta$, then $P(a+\delta) \neq 0$.

Now let $\Delta$ be the lub of the set of $\delta$ 's such that $0<\delta<b-a$ and for which (5) holds on $(a, a+\delta)$. Clearly (5) holds on $(a, a+\Delta)$, and so (by the above argument) (5) holds at $t=a+\Delta$. Unless $\Delta=b-a$, the continuity of $P(t)$ gives a contradiction to the lub property of $\Delta$. This completes the proof.

The following theorem, corresponding to Theorem A, is now almost immediate.

THEOREM 1. If (II) holds for products excluding the $a_{i i}(t)$ 's, the problem (3) has a unique solution.

Proof. The proof depends only on Lemma 3, and so we must show that Lemma 3 holds even without condition (0). To this end, let $z(t)=G(t) x(t)$, where $x(t)$ satisfies (III) and $G(t)$ is the diagonal matrix for which $g_{i i}(t)=\exp \int_{a}^{t}-a_{i i}(s) d s$. Then $z^{\prime}=B(t) z$, where $b_{i i}(t) \equiv 0$ and, for $i \neq j, b_{i j}(t)=a_{i j}(t) \exp \int_{a}^{t}\left[a_{j j}(s)-a_{i i}(s)\right] d s$. Thus if (II) holds for 
$A(t)$ then (II) holds for $B(t)$, with the same $s_{i N}$ 's; if (III) holds for $x(t)$ then (III) holds for $z(t)$; and (0) holds for $B(t)$. Thus Lemma 3 as it stands applies to $z(t)$, and also, since $x_{i}(t)=z_{i}(t) \exp \int_{a}^{t} a_{i i}(s) d s$, to $x(t)$. Thus condition ( 0$)$ can be eliminated from Lemma 3 , and the proof is complete.

The theorem corresponding to the dual of Theorem A is contained in the following statements. Corresponding to (I), (II), and (III) we have:

(I') As (I), except that the products with an odd number of factors and those with an even number of factors differ in sign, and there is at least one product, say with $r$ factors $\left(r \leqq m_{K}\right)$, which is nonzero at $b$. Let $(-1)^{r} s_{K N}^{\prime}$ be 1 or -1 according as this last product is positive or negative at $b$. Let $s_{N N}^{\prime}=1$.

(II') For some fixed $N$, (I) holds for each $K \neq N(1 \leqq K \leqq n)$; $a_{N K} s_{K N}^{\prime} \leqq 0$; and the $m_{K}$ 's may all be taken equal.

$\left(\mathrm{III}^{\prime}\right) x^{\prime}=A x$, and $x_{i}(b)=\delta_{i N}(1 \leqq i \leqq n)$.

Lemma 2 '. If (0), ( $\left.\mathrm{I}^{\prime}\right)$ and $\left(\mathrm{III}^{\prime}\right)$ hold, there is a $\delta>0$ such that $s_{K N}^{\prime} x_{K}(t)>0$ on $(b-\delta, b)$.

Proof. The proof is like that of Lemma 2, using (4). Alternatively, let $A(t)=A(b)$ for $t>b$; let $s=2 b-t, B(s)=-A(t)$, and $w(s)=x(t)$; and apply Lemma 2 directly to the system $w^{\prime}(s)=B(s) w(s)$ on the interval $[b, 2 b-a]$.

Lemma $3^{\prime}$. If (0), ( $\left.\mathrm{II}^{\prime}\right)$ and $\left(\mathrm{III}^{\prime}\right)$ hold, then

$$
s_{i N}^{\prime} x_{i}(t)>0 \quad(1 \leqq i \leqq n)
$$

holds on $[a, b)$.

ThEOREM 1'. If (II') holds for products excluding the $a_{i i}(t)$ 's, the problem

$$
y^{\prime}=A y+f, \quad Q y(a)+P y(b)=\beta
$$

has a unique solution.

Theorem 1 implies a stronger version of Theorem A, since the hypotheses of Theorem A imply (II) for each $N$. Further, coefficient matrices with vanishing entries can be treated; in particular, known theorems (e.g., see [2] and [3]) for the $n$th order scalar case are implied.

3. The three-point problem. Let $a<c<b$.

Theorem 2. Let $M$ and $N$ be fixed $(1 \leqq M \leqq n, 1 \leqq N \leqq n, M \neq N)$. 
Let (II) hold on $[c, b]$ and (II') hold on $[a, c]$ for products excluding the $a_{i i}(t)$ 's, for $N$ and also for $M$, with $s_{M N} s_{N M}^{\prime}=-1$. Let $R=\left(\delta_{i M} \delta_{j M}\right)$, $Q=\left(\delta_{i N} \delta_{j N}\right)$, and $S=E-R-Q$. Then the problem

$$
y^{\prime}=A y+f, \quad Q y(a)+S y(c)+R y(b)=\beta
$$

has a unique solution.

Proof. Let $X^{\prime}=A X, X(c)=E$; it suffices to show that $Q X(a)$ $+S X(c)+R X(b)$ is nonsingular, the determinant in question being $x_{N N}(a) x_{M M}(b)-x_{N M}(a) x_{M N}(b)$. By Lemma $3, x_{M M}(b)$ and $s_{M N} x_{M N}(b)$ are positive; by Lemma $3^{\prime}, x_{N N}(a)$ and $s_{N M}^{\prime} x_{N M}(a)$ are positive; hence the determinant is positive.

It is a matter of detail to verify that the hypotheses of Theorem B imply those of Theorem 2. As in the two-point case, coefficient matrices with vanishing entries, and in particular the scalar case (e.g., Theorem 2 in [2]), are allowed.

\section{REFERENCES}

1. J. B. Garner and L. P. Burton, Solutions of linear differential systems satisfying boundary conditions in the large, Proc. Amer. Math. Soc. 12 (1961), 100-106.

2. J. B. Garner, Boundary conditions for the linear differential equation, Amer. Math. Monthly 69 (1962), 47-50.

3. W. Leighton and Z. Nehari, On the oscillation of solutions of self-adjoint linear differential equations of the fourth order, Trans. Amer. Math. Soc. 89 (1958), 325-377.

UNIVERSITY OF UTAH 